\section{EN CASA CON MI BEBÉ. CUIDADO INFANTIL EN SECTORES MEDIOS PROFESIONALES URBANOS}

\author{
AT HOME WITH MY BABY. \\ CHILD CARE IN PROFESSIONAL URBAN SECTORS
}

PABLO DE GRANDE ·

Instituto de Investigación en Ciencias Sociales -

Universidad del Salvador - Consejo Nacional

de Investigaciones Científicas y Técnicas

(IDICSO-USAL-CONICET)

pablodg@gmail.com

\section{Resumen}

El artículo realiza un estudio del cuidado de niños menores de un año en sectores medios profesionales de la Ciudad de Buenos Aires. Para esto, se analizan las actividades, interacciones y sentimientos observados en cuatro horas de cuidado entre una mamá y su bebé de 11 meses de edad, en el contexto de una serie de entrevistas dirigidas a madres y padres de este estrato socioeconómico. El trabajo se enmarca en los estudios sociales del cuidado y la sociología de las emociones, campos que han buscado comprender mejor las motivaciones y vivencias de los actores sociales en sus prácticas afectivas con otros. Como hipótesis principal se plantea que las dinámicas y ritmos del cuidado en sectores medios pueden asumir organizaciones temporales singulares, ligadas a la simultaneidad, la alternancia, el vínculo continuo y la disponibilidad permanente entre los niños y sus cuidadores.

\section{Descriptores}

emociones / primera infancia / crianza / cuidados / profesionalización
ESTUDIOS SOCIALES

revista universitaria semestral ·

año XXXI $\cdot n^{\circ} 60 \cdot$ Santa Fe Argentina

Universidad Nacional del Litoral ·

enero-junio $\cdot 2021$ [ ISSNe 2250-6950 ]

DOI [ 10.14409/es.v60i1.8283 ] PP [ 97-124]

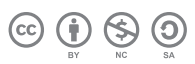

\section{Abstract}

The paper studies the care of children $<1$ year in professional sectors of the City of Buenos Aires. The activities, interactions and feelings observed in four hours of care between a mother and her 11-month-old baby are analyzed in the context of a series of interviews carried out with mothers and fathers of this socioeconomic stratum. The project is part of the social studies of care and the sociology of emotions, fields that have sought to better understand the motivations and experiences of social actors in their affective practices with others. The main hypothesis is that the dynamics and rhythms of care in this sector can assume unique temporal arrangements, linked to simultaneity, alternation, continuous bonding and permanent availability among children and their caregivers.

\section{Describers}

emotions / early infancy / childreading / care / profesionalization

Recibido: 21 / 08 / 2019 Aprobado: 16 / 07 / 2020 


\section{INTRODUCCIÓN}

El análisis que se presenta en este artículo busca dar cuenta de los procesos que acompañan el despliegue, material y simbólico, de estar al cuidado de un niño pequeño, en pos de comprender la lógica temporal, relacional y emocional que este tipo de cuidado puede suponer o configurar.

La bibliografía sobre sectores medios urbanos modernos afirma que es infrecuente que un adulto con estudios universitarios haya dedicado una cantidad de tiempo significativa a tareas de cuidado durante su infancia o su juventud ${ }^{1}$ (HAGESTAD Y UHLENBERG, 2005; BRONFENBRENNER, I982; ROGOFF, I98I). Esta clausura, aunque matizada por género, da cuenta en los estilos de vida urbanos y modernos de una separación tanto de hombres como de mujeres de los procesos vitales y familiares. El cuidado de bebés aparece estilizado como un juego infantil-femenino, dando un carácter sexuado y abstracto a una práctica que en otros contextos sociales es cotidiana y frecuente. En éstos, en cambio, la mayor parte del tiempo diario de la infancia y la juventud se encuentra destinado a la formación educativa y — desde la mayoría de edad - al tránsito por ámbitos laborales.

En las entrevistas hechas en mi investigación sobre bebés de sectores medios en la ciudad de Buenos Aires, dos fenómenos eran llamativos respecto a cómo se percibía la temporalidad del cuidado de sus hijos pequeños.

Por una parte, cuando el cuidado del bebé durante el día dependía de la presencia de la madre en la casa, esto era prontamente explicado como un impasse en su carrera profesional. La convivencia exclusiva — ligada a licencias laborales o a dedicaciones horarias parciales - era vista como un estado de excepción. Más que ante un cambio de vida ya completado, se estaba frente a un período transicional entre la vida laboral previa y una futura, la cual ocurriría a la par de la maternidad y sería en cierto modo una combinación de la actual y aquella pasada.

1] Dice Uri Bronfenbrenner: «Es ahora posible para una persona de 18 años graduarse en una escuela secundaria estadounidense sin haber nunca tenido que hacer una tarea de la que otro dependiera. Igualmente desastroso desde esta misma perspectiva, es posible para un joven, tanto mujer como varón, graduarse de la escuela secundaria o la universidad sin nunca haber sostenido un bebé en sus brazos por más de unos segundos, sin nunca haber tenido que cuidar a alguien que fuera viejo, que estuviera enfermo o solo, sin nunca haber tenido que dar consuelo o ayudar a otro ser humano que realmente lo necesitara» (BRONFENBRENNE, 1982: 118, trad. propia). 
Por otra, cuando las madres se refirieron a los días en que estaban con sus bebés en sus hogares, fue recurrente la aparición de un extrañamiento en la percepción del uso del tiempo tal como era visto por las entrevistadas. En las charlas, varias de ellas manifestaron sentir que "no hacían nada» y que, a la vez, el tiempo y las fuerzas con frecuencia no alcanzaban, lo que hacía de los días algo extendido y extenuante. El tiempo escaseaba, y a la vez, no parecía ser usado en ninguna cosa.

Estas dos tensiones — la provisionalidad y el carácter escurridizo del tiempoparecían exceder las discusiones sobre cuidado y temporalidad tratados en los debates sobre la doble carga de mujeres que alternan entre sus tareas laborales y domésticas (HOCHSCHIL y MACHUNG, 1989) y las pujas de poder al interior de los hogares (GUTIÉRREZ SASTRE, 2002; COVARRUbIAS FEREGRINO, 20I8). Asimismo, si bien esto ocurría sobre el telón de fondo de la maternalización del cuidado (FLORES ÁNGELES Y GUERRERO, 2OI4; NARI, 2004) su ocurrencia no resultaba completamente explicable por el modelo de roles derivado de la misma (CASTAŃEDA RENTERÍA, 2OI9; MUÑIZ GALLARDO, Y RAMOS TOVAR, 20I9). ¿Qué ocurría en esas horas de cuidado? ¿Qué hacía dificultoso el registro de ese hacer y de ese estar? ¿Por qué el bebé, siendo un nuevo miembro estable de la familia, aparecía ligado a arreglos provisorios de cuidado?

El paso a la observación, a situarse junto a la actividad de cuidado, podía dar una posición privilegiada para ayudar a poner en evidencia aquello que se había manifestado en forma oscura, vaga, contradictoria, incluso si consistía en algo cotidiano y conocido. Para esto, dispusimos con una de las familias entrevistadas la revisita al hogar para realizar una observación de 4 horas que produjera un registro fotográfico del tiempo de cuidado y del estar de la mamá y su bebé.

Para presentar estos resultados, en primer lugar, en la siguiente sección se encuadran estas preocupaciones en la bibliografía de la experiencia cotidiana del cuidado y las emociones. En segundo lugar, se introducen los criterios por los cuales se produjo el material empírico, así como la estrategia de análisis por medio de la cual se organizaron las dimensiones observadas. Luego, se presentan las secuencias reconocidas en la observación. Finalmente, se realizan algunas reflexiones sobre estas prácticas y las especificidades que pudieron reconocerse a partir del análisis y de los resultados obtenidos. 


\section{EL CUIDADO INFANTIL Y LAS EMOCIONES}

Desde la década de 1980, la mirada sobre las prácticas sociales y las experiencias cotidianas ha ganado espacio en las agendas de investigación social. En oposición a marcos de corte más normativista y macro-social, crecieron líneas de trabajo que conciben sus estrategias metodológicas y sus modelos explicativos considerando el carácter situado de los actores, la dimensión corporal y práctica de la experiencia y en combinación de ambas, el nivel emotivo de las prácticas sociales (sCRIBANO, 2OI2; LUNA ZAMORA, 2000; THOITS, 1989).

Estas producciones académicas dieron lugar a una serie de nuevos objetos y miradas antes menos presentes en investigación social, deviniendo el cuerpo (SHILLING, 2003; SHEETS-JOHNSTONE, I990), la interacción (POWELL Y DÉPELTEAU, 2OI3) y la afectividad (ARIZA, 2OI5; TICINETO CLOUGH Y HALLEY, 2007; TENOUTEN, 2006) campos de abundante desarrollo teórico y empírico ${ }^{2}$.

Si bien la sociología clásica señalaba a la acción emotiva y a las acciones «no racionales» (BOUDON, I98I: I8) como claves de análisis necesarias de lo social (ARIZA, 2OI6: IO; CHAHBENDERIAN, 2OI3; DETTANO, 2OI3; LUNA ZAMORA, 2000), los recientes estudios del campo de las emociones han sido más decididos en dar una atención explícita a los compromisos y dinámicas de la afectividad (THOITs, 1989: 317). A partir de ellos, podemos encontrar desde mediados de la década de I970 teorías específicas producidas para dar cuenta de fenómenos emocionales reconocidos como dimensión central de las dinámicas sociales (TENOUTEN, 2006; BERICAT ALASTUEY, 2000; HOCHSCHILD, 1975).

Estos cambios ocurrieron en diálogo con la producción de los estudios feministas que abogaron —entre otras cosas - por visibilizar esferas de la actividad humanas relegadas por sesgos patriarcales, industrialistas y etnocentristas (FLORES Y TENA GUERRERO, 2OI4; MAFFÍA, 2007; FISHER Y TRONTO, I99I; SEGATO, 2OI8). Lo doméstico, lo emotivo y lo infantil aparecieron como instancias históricamente desplazadas de la producción hegemónica de conocimiento social.

2] Parcialmente, los estudios ligados a las emociones y su relación con la vida social se vinculan con indagaciones que en psicología social se habían emprendido en forma sostenida desde la posguerra en torno a la relación entre conducta, emociones y subjetividad con diversos abordajes metodológicos (BLOCK, 1957; ALLPORT, [1954] 1977; MILGRAM, 1963). Estos estudios sociales de las emociones se diferenciaron al mismo tiempo con otra parte del campo psicológico (el evolucionista), al negar un anclaje entre el estudio de las emociones y el largo camino de la evolución psicobiológica y optar por una mirada constructivista/cultural de lo emocional (PRINZ, 2004). 
Estas preocupaciones, a su vez, guardan estrecha relación con el campo de producción académica relativamente reciente de los estudios del cuidado. Joan Tronto y Carol Gilligan fueron autoras centrales en esta transformación del tratamiento académico del cuidado, al reformularlo a partir de dos hechos decisivos del cuidar. Según estas autoras, el cuidado debe ser considerado como una disposición ética y necesaria de ayuda al prójimo y, a la vez, resaltarse el hecho de que el cuidado ha sido históricamente asumido por mujeres (GILLIGAN, 1982: 17; 20I3; TRONTO, I993, 2008, 2013). Este nuevo abordaje al cuidado ha sido considerado como «una teoría filosófica del ser solícito, de la preocupación por el otro o de la atención puesta en otro" (PETIT, 20I3).

Dos casos de investigaciones que han rastreado cuestiones ligadas a la experiencia y la emotividad del cuidado infantil en la Argentina son la investigación de Eleonor FAUR (20I2) donde recupera los temores que acompañan a las decisiones vinculadas a los arreglos familiares de cuidado en madres del barrio de La Boca en la ciudad de Buenos Aires, y el trabajo de María Adelaida Colángelo (2006) en su investigación sobre decisiones ligadas a la salud en la ciudad de La Plata, donde da cuenta de las percepciones de madres de clase media con bebés pequeños ${ }^{3}$. Sin embargo, las emociones no han sido puestas en el centro de estos análisis (sí los criterios en las tomas de decisión), y no se han ocupado específicamente del primer año de cuidadó .

En el caso de Colángelo, quien también aborda sectores medios urbanos, la soledad frente a los procesos de cuidado y crianza aparece como un sentimiento recurrente entre estas madres. En mi investigación, la configuración residencial y relacional pareció explicar buena parte de la sensación de aislamiento que experimentaban las madres con sus bebés en los primeros meses de cuidado (DE GRANDE, 2OI5; DE GRANDE, 20I6).

3] En el relevamiento hecho por Scribano y equipo (SCRIBANO, 2015) sobre investigaciones dedicadas al campo de las emociones en la Argentina, los estudios más próximos al campo de los cuidados lo representan aquellos relativos a los cuidados en salud, sin registrarse allí trabajos ligados a la emotividad en espacios doméstico con niños.

4] En este sentido, la caracterización de las interacciones madre-bebé y el contexto de cuidado han sido exploradas con mayor detenimiento en otros contextos nacionales y culturales, como se detalla en ABELS, KELLER, PRERNA, HINA, JIGISHA, SHRUTI, SUCHETA Y ARUNA (2005). 
El departamento familiar-nuclear, con estilo prolijo y luminoso, de pocos ambientes, relativamente distante de tíos y abuelos, separado en altura de la calle y del vecindario como espacios de sociabilidad instantánea, era el modo predominante en que los niños y sus madres habitaban. Esta disposición de vivienda se asociaba a su vez con un conjunto de objetos «diseñados para bebés» que situaban al bebé en el centro de la escena: cuna, mamadera, juguetes, libros para bebés, vasos y platos infantiles (DE GRANDE, 2OI6).

Las redes interpersonales que unen a los hogares con otros hogares, por su parte, dieron allí cuenta de una baja disponibilidad de otros adultos durante las horas del día. Amigos y amigas, personas del círculo familiar extenso, producto de sus propias inserciones profesionales, no contaban con poder acercarse durante la semana a compartir tiempo con la madre o su bebé, a tomar «mates», a dar una mano, a charlar (DE GRANDE, 2OI5). Los encuentros con ellos quedaban restringidos a visitas esporádicas en los fines de semana o a coincidir en los festejos de cumpleaños, bautismos y similares.

En este marco, se avanzará en este artículo en explorar la intersección del cuidado con la emotividad en la convivencia con bebés, haciendo énfasis en su relación con las dinámicas prácticas e intersubjetivas de la cotidianeidad.

\section{METODOLOGÍA}

\section{PARTICIPANTES Y PROCEDIMIENTO}

Como se adelantó anteriormente, para la elaboración del material empírico de este artículo se puso como objetivo hacer observación de una mañana en el domicilio de uno de los bebés que formaban parte de una muestra de entrevistas en la Ciudad de Buenos Aires. Esta muestra estaba conformada por I4 familias con bebés a las que se había visitado previamente, cuyas madres tenían estudios universitarios y sus bebés eran menores a I2 meses de edad. La elección del caso fue intencional. Martina al momento de la entrevista tenía in meses de edad, y su madre 32 años.

Durante la observación se realizó un registro fotográfico que constituyó el material de campo central de este artículo.

El primer contacto con el espacio cotidiano de Martina (la bebé) había sido establecido un mes antes, en el cual se había compartido una mañana con la madre y con la niña, charlando bajo la modalidad de entrevista acerca de las rutinas, 
cuidados diarios y organización general de la vida cotidiana que había tenido lugar desde su nacimiento.

El material fue producido entre las 9:30hs y las 13:30hs de un día viernes, en la vivienda del barrio de Villa Urquiza donde ambas residen junto al papá de Martina. En ese intervalo se tomaron fotos de las actividades que involucraban a Ana y a su hija Martina ${ }^{5}$ totalizando un registro de 240 imágenes.

En este sentido, las condiciones de habitabilidad, la situación de edad, posición socioeconómica, historia de salud/enfermedad y la estructura familiar guardaban suficientes semejanzas con las demás entrevistadas como para habilitar a la realización de una observación más extendida considerando a este caso como correspondiente al grupo más general de madres de sectores medios profesionales visitadas previamente.

\section{LA SECUENCIACIÓN EN EL ANÁLISIS}

El análisis realiza un recorrido por la serie de acontecimientos que tuvieron lugar en las horas de observación. Al mismo tiempo, busca recuperar parcialmente los sentidos que estos acontecimientos cobraron para Ana y Martina; cómo esa mañana se sucede y se sostiene por la interpretación activa que cada una de ellas hace de los hechos y por los afectos, valores y objetivos que enlazan a aquello que perciben y manifiestan.

La segmentación temporal del análisis se apoya en las nociones de secuencia y de estructura secuencial, de Jean Michel Adam. Este autor sugiere que los sentidos de un relato no se inscriben en un marco homogéneo, sino que por el contrario responden a metas, repertorios y lógicas adscriptas a secuencias reconocibles. Estas secuencias dan claves para interpretar y orientar la acción, y gozan de una relativa cohesión semántica interna (ADAM, I992: 2I; SÁNCHEZ MARTÍNEZ, 2002: 4)

En el análisis, el corpus de 240 imágenes fue utilizado para realizar el reconocimiento temporal de las secuencias significativas que ocurrieron durante la observación. Tomando en cuenta las actividades que podían reconocerse en las imágenes, se estableció una lista de secuencias, determinando su inicio y duración a partir de la hora de registro de cada fotografía.

5] Se han asignado nombres ficticios para la presentación de resultados. 
En segundo lugar, las imágenes fueron utilizadas para construir un subconjunto de 42 fotografías con el objeto de presentar un registro visual de las disposiciones corporales, gestuales y del contexto en que las secuencias ocurrieron.

\section{LOS ELEMENTOS DE LA CONDUCTA}

Por su parte, cada una de las secuencias será analizada en su contenido a partir de tres elementos conceptuales para sistematizar su abordaje. Estos tres elementos siguen la propuesta organizativa de George Homans para la descripción de escenas sociales, y son: la actividad, la interacción y el sentimiento (BERICAT, 20I2: 7; CRUZATA SANTOS, 2005; HOMANS, I970).

En primer lugar, el concepto de actividad se introduce como un elemento para agrupar aspectos del orden del hacer (HOMANs, I970, p. 62). En el análisis retomaremos este elemento como un aspecto en que se despliega el cuidado y el estar con un niño, describiendo a ambos (madre y bebé) como sujetos inmersos en diferentes cursos de acción a lo largo de la observación.

En segundo lugar, el concepto de interacción es introducido por Homans para señalar los elementos de la conducta social en donde con independencia de la actividad particular que se despliegue, puede notarse un nivel de comunicación o de interdependencia que la trasciende. El volverse compañero, el tomar contacto, el participar, dice Homans, son manifestaciones del elemento interaccional de la conducta grupal que no dicen nada en particular del contenido de dichos contactos y participaciones, sino que seńalan el refuerzo o la existencia de un vínculo interpersonal, de dos o más personas (HOMANs, I970, p. 64).

Finalmente, Homans incorpora como un tercer elemento el nivel del sentimiento. Con ello repara en aquello que por miedo, simpatía, respeto, melancolía, hambre, afecto, entre muchos otros sentimientos posibles, matiza o resalta las percepciones internas de una vivencia. Para dar cuenta de ellos, dice Homans, es preciso muchas veces apoyarse en el entrenamiento cotidiano, en la práctica corriente, por la cual solemos reconocerlos a través de indicios visibles: leves cambios de tonos de voz, expresiones en el rostro, movimientos en las manos, formas de mover el cuerpo (HOMANS, 1970, p. 66).

Este modelo de análisis proporciona una herramienta heurística para la descripción de series de eventos protagonizados por una o varias personas. Sus elementos 
son concebidos como co-ocurriendo en un todo de acción: las actividades se inician, configuran y desarrollan en diálogo con el sentir. La interacción, por su parte, provoca y se apoya en el nivel de la experiencia y en su emotividad, a la vez que la actividad y la interacción se estructuran mutuamente, atravesadas en su sentido y en sus posibilidades materiales por el plano de la afectividad. Consecuencia de esta imbricación, la organización del análisis en estas tres categorías busca ayudar a una descripción de las secuencias, sin por esto sugerir una existencia independiente de estos niveles.

\section{RESULTADOS}

El análisis condujo a la identificación de II secuencias dentro de las 4 horas de observación, que constituyeron el primer resultado del mismo (su secuenciación y su cantidad), explicitando que el tiempo de cuidado difícilmente podía ser visto como una tarea continua y homogénea.

Estas secuencias cubrieron la totalidad del tiempo de observación, y se destacaron por la alternancia de sentimientos, sentidos, cadencias en la acción y formas de organización, así como por la variación en la función, la afectividad y el sentido del movimiento, la palabra y la mirada tanto por parte de la niña como de su mamá.

\section{Preparación para el desayuno - 9:30 a 9:50}

La primera secuencia involucra la llegada del investigador a la casa, momento en el cual Ana y Martina ya habían iniciado su día. El padre había salido a trabajar más temprano, hacia una oficina de la zona céntrica de la ciudad. Martina estaba en el living, al igual que Ana, y se inició así el registro fotográfico.

Ana comentó que este rato, en el cual Martina estaba en el corralito y miraba algunos programas de televisión, le permitía a ella comenzar a avanzar algunas cuestiones domésticas, tales que poner lavados de ropa, verificar comida disponible, ordenar juguetes, ropa u otras cosas sueltas en la casa (Figura I).

Actividad: La actividad se desdobla para Ana y Martina en dos ocupaciones diferenciadas. La realizada por Ana se caracteriza por el ir y venir por la casa, disponiendo algunas cosas en la mesa del living, revisando la heladera para detectar faltantes para el almuerzo y comidas posteriores, poniendo a hacer un lavado de 
ropa. En el caso de Martina, la actividad es de tipo más estático, con una movilidad parcialmente limitada dentro del corralito, en la que reparte su atención entre tomar y soltar objetos y mirar las escenas en la televisión de la Doctora Juguetes.

FIGURA 1.

LA ACTIVIDAD COMIENZA EN EL LIVING DEL DEPARTAMENTO PREPARANDO EL DESAYUNO

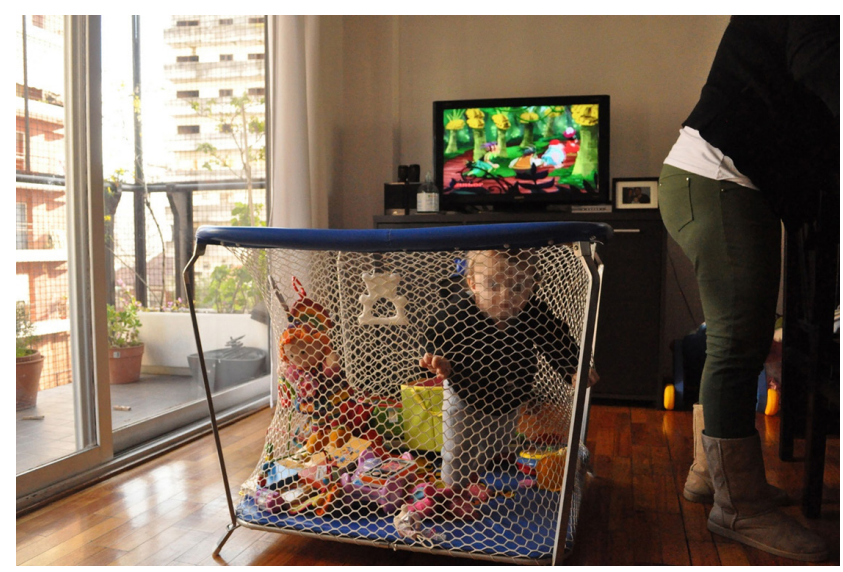

FUENTE: elaboración propia en base a observación de campo.

Interacción: Entre Ana y Martina tiene lugar en esta etapa una aparente desconexión, en función de que ambas realizan actividades diferenciadas. Sin embargo, la atención que se dedican es recíproca: Martina observa con interés el ir y venir de Ana; Ana mantiene una mirada intermitente sobre Martina, para verificar que no ocurran incidentes y que no haya un malestar en Martina que deba ser atendido. A la vez, intercambian miradas, la mamá dice frases en baby talk que renuevan el interés de Martina y le destaca el sonido de algún juguete u objeto del corralito para dirigir su atención.

Sentimientos: El ritmo de esta secuencia viene principalmente dado por el pulso de la rapidez. La sensación principal es el apremio, dado por la combinación de una diversidad de cosas para ordenar y preparar para el día con la limitación manifestada por Ana respecto a que Martina solo se entretiene un tiempo corto en forma autónoma con los juguetes del corralito y la televisión. 


\section{Cambio de ropa - 9:50 a 9:55}

La siguiente secuencia interrumpe la primera por la aparición progresiva, pero finalmente generalizada, de saliva sobre la ropa de Martina. Al estar gran parte de su campera humedecida por la baba, Ana decide detener sus actividades para proveer de ropa seca a Martina (Figura 2).

Actividad: el cambio de ropa conduce a Martina hasta su habitación. Llega a ella en brazos de su madre, quien le quita la campera húmeda. Mientras Ana elige una nueva campera, Martina espera dentro de la cuna, en la que normalmente duerme. Como Martina está dando sus primeros pasos, vuelve desde la pieza hasta el living caminando, tomada de Ana.

\section{FIGURA 2.}

ANA ADVIERTE QUE LA ROPA DE MARTINA TIENE SALIVA EN EXCESO Y PROCEDE A CAMBIARLA.

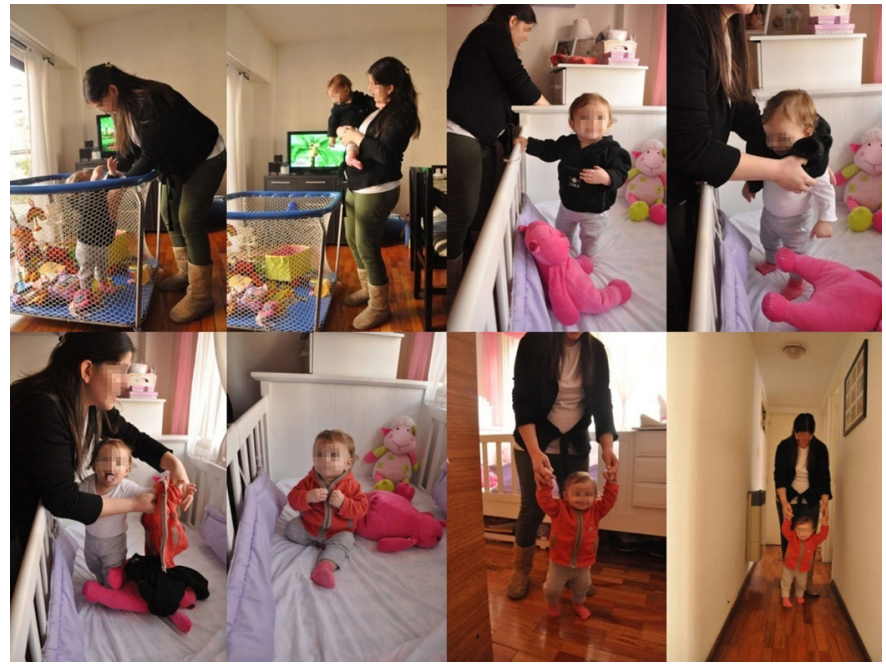

FUENTE: elaboración propia en base a observación de campo.

Interacción: a diferencia de la secuencia anterior, el contacto directo es casi constante, dando apoyo Ana a Martina para el transporte en brazos hacia la habitación, para el cambio de ropa y para el regreso a pie. En estas tres modalidades 
(alzada, cambio de ropa y soporte al caminar) la interacción se presenta por medio de movimientos coordinados y conocidos.

Sentimientos: Ana desarrolla la actividad con un tono corporal que combina lo afectuoso y con lo maquinal, correspondiéndole Martina con un comportamiento similar. A simple vista, la secuencia parece no resultarles ni problemática ni novedosa, por lo que la transitan con parcial indiferencia.

\section{Mamadera - 10:05 a 10:15}

La tercera secuencia tiene inicio luego de unos pocos minutos tras el cambio de ropa. Martina toma su mamadera, habiendo la madre completado la secuencia de orden de la casa que había iniciado en la primera secuencia (Figura 3).

Actividad: Martina toma la mamadera, sentada sobre la falda de Ana quien la sujeta con sus manos. La escena se concentra en una de las sillas junto a la mesa del living, y la madre sostiene la mamadera hasta que ésta se acaba. La toma se sucede en un solo paso, es decir, no surgen interrupciones a la misma ni Martina la aleja de su boca hasta el momento de finalizada la leche.

Interacción: en la interacción que se deriva de

FIGURA 3.

MARTINA TOMA LECHE DE LA MAMADERA SOSTENIDA POR SU MADRE.

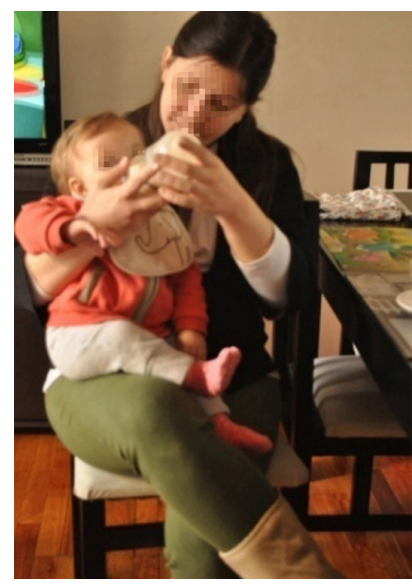

FUENTE: elaboración propia en base a observación de campo. esta secuencia, Martina se mantiene más pasiva que en las anteriores, y aunque participa de la acción tomando la leche, su cuerpo está mayormente quieto y reposa sobre Ana, quien toma la responsabilidad general de la acción, incluyendo el velar por que la mamadera sostenga la inclinación correcta, y que Martina se mantenga en forma segura sobre su falda.

Sentimientos: si bien Martina es la receptora de la leche en torno a la cual esta secuencia se organiza, el carácter pautado de la alimentación no la pone como principal demandante del proceso. Toma la mamadera de buen grado, pero sin muestras previas de hambre, por lo que el matiz afectivo que la secuencia expresa se organiza en torno al atender una actividad rutinaria, antes que el dar respuesta a una necesidad corporal tal que el hambre y su satisfacción. 


\section{Jugando - 10:15 a 10:40}

Completada la toma de la mamadera, Ana coloca algunos juguetes fuera del corralito para dejarlos a disposición de Martina. Estando estos en el suelo y en el sillón del living, la bebé comienza a desplazarse para hacer uso de ellos y empieza a jugar (Figura 4).

Actividad: la madre circula poniendo en su sitio diversas cosas de la casa, mientras que Martina juega por sus propios medios. Sin embargo, el acompañamiento de la madre es frecuente, y la autonomía de Martina es parcial. Ana le alcanza objetos que se alejan más de lo esperado, cuida los ejercicios de caminata y seńala juguetes o juegos desatendidos por Martina para devolverlos a escena.

FIGURA 4.

COMIENZA LUEGO DEL PRIMER DESAYUNO EL PERÍODO DE JUEGO FUERA DEL CORRALITO.

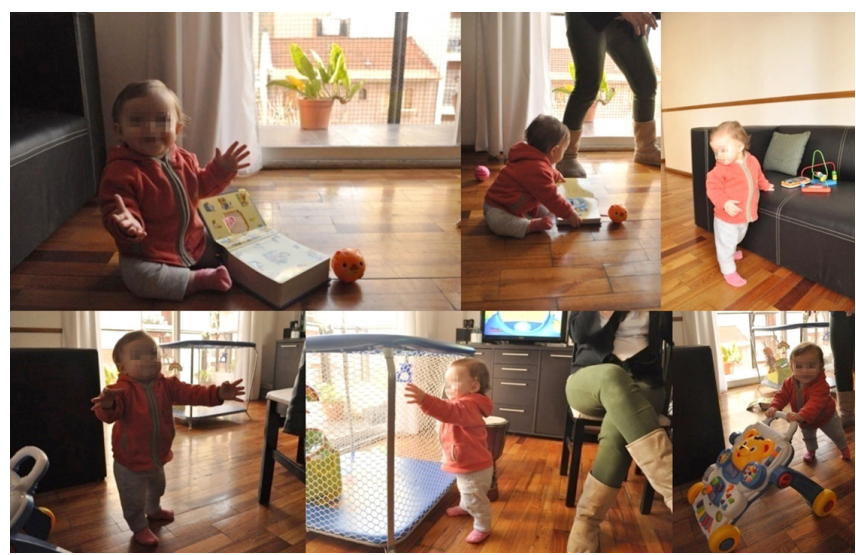

FUENTE: elaboración propia en base a observación de campo.

Interacción: en el desarrollo de las actividades se da una forma de interacción por la cual Martina establece juegos individuales, que elije por sí misma, para los que sin embargo cuenta con la mirada y el cuidado de su madre, en tanto no siempre puede hacer plenamente uso de ellos sin su ayuda. Ana actúa en la anticipación del riesgo y en el apuntalamiento de las acciones de Martina. 
Sentimientos: si bien la madre se encuentra atenta y ocupada a la vez, el ánimo es más bien alegre y aprovecha para conversar y señalar al investigador los tipos de juego con los que Martina disfruta más en las últimas semanas. Martina se desplaza por el living y alterna entre diferentes juguetes (libros, pelotas, andador), los cuales le resultan en su mayoría atractivos y ganan cada uno su atención por varios minutos.

\section{Desayuno (parte sólida) - 10:40 a 11:05}

Luego de un momento de juego, la madre sirve un té al investigador y se incorpora a la mesa, invitando también a Martina a compartir comida sólida para completar el desayuno antes iniciado con la mamadera (Figura 5).

Actividad: Martina se incorpora a la mesa junto con la madre y el investigador. Se sienta en una silla, en la que se mantiene estable sin ayuda de la mamá. En la mesa hay comida puesta a disposición, la cual toma, manipula y come en forma autónoma.

FIGURA 5.

SOBRE UNA SILLA, MARTINA COME UNA PORCIÓN DE BUDÍN.

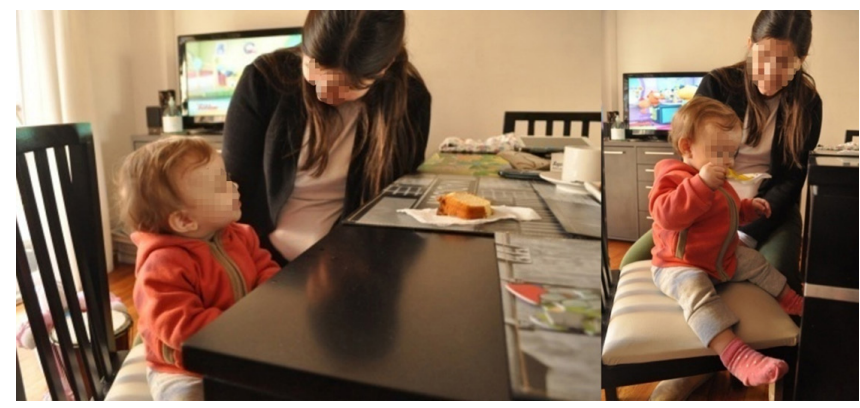

FUENTE: elaboración propia en base a observación de campo.

Interacción: en esta secuencia la atención entre Ana y Martina se sostiene, aunque funcionalmente actúan con mayor independencia que en las demás comidas que se sucedieron durante la observación. El intercambio se centra en miradas y comentarios, y en el cuidado de que Martina no caiga de la silla ni malogre invo- 
luntariamente la comida. Ana, Martina y el investigador comparten la acción en cierto pie de igualdad, tomando un té y comiendo porciones de budín.

Sentimientos: la mayor independencia concedida a Martina coincide con un clima de mayor distención, en el cual la madre conversa alternadamente con la bebé y con el investigador. Martina disfruta de la comida, la cual en parte se deshace a la presión de su mano pero llega también a su boca y logra probarla y comerla con interés.

\section{Siesta - 11:05 a 11:10}

Luego del desayuno, Martina empieza a mostrar señales de hastío. Si bien brevemente regresa al juego, su madre identifica en estos signos la necesidad de dormir la siesta de media mañana. Aunque Ana afirma que el dormir en brazos no es una práctica habitual, ante el ánimo crecientemente turbado de la bebé, decide alzarla (Figura 6).

Actividad: la acción de conducir a Martina hacia la siesta es relativamente breve, adormeciéndose la niña a los pocos minutos de llegar a los brazos de su madre. Ana mantiene a Martina en brazos mientras la mece, para luego conducirla hasta la pieza, colocarla en la cuna y arroparla.

\section{FIGURA 6.}

EN BRAZOS DE SU MADRE, MARTINA SE DUERME Y QUEDA ACOSTADA EN SU CUNA.

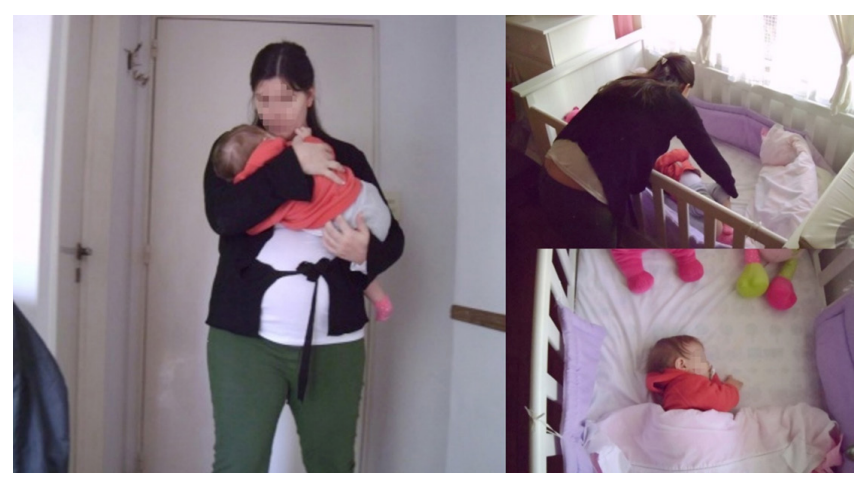

FUENTE: elaboración propia en base a observación de campo. 
Interacción: el rol de Ana es activo en este proceso, siendo central en permitir a Martina conciliar el sueño. Martina, en la interacción, se muestra complacida por el aumento de proximidad entre ambas, y se deja llevar por el arrullo de su madre.

Sentimientos: en esta secuencia madre e hija pasan por estados anímicos diferenciados. Martina atraviesa una serie cambiante que comienza con el hastío producido presumiblemente por el sueño en aumento, y logra un estado de placidez y sueño hacia el final. Ana en cambio varía en menor medida su ánimo con relación a la situación, si bien gana también algo de tranquilidad al poder guiar a Ana hacia su descanso y dejarla dormida en la habitación.

\section{Despertar - 12:10 a 12:15}

Cabe aclarar dos razones por las que no se ha introducido una secuencia para lo acontecido mientras Martina dormía, lo que ocurrió entre las ir:IO y las I2:IO.

Por una parte, las secuencias comprenden las actividades de interacción entre la niña y su madre, así como los sentimientos de ambas, por lo que esta secuencia de siesta hubiera introducido un caso diferenciado, en tanto Martina no realizó acciones diferentes al mero dormir durante esa hora. Por otra, en este lapso Ana conversó con el investigador casi en su totalidad. Sin desconocer o desestimar la influencia de la presencia del investigador en las demás secuencias, ésta en particular no parecía aportar desde su contenido a los eventos de cuidado diario con la bebé.

Damos paso por ende a la secuencia referida al despertar de Martina de su siesta (Figura 7).

Actividad: al oírse ruidos en la habitación de Martina, su madre asiste para poder ayudarla a reincorporarse. Al llegar a la pieza vemos a Martina de pie en la cuna, con su chupete en la boca, esperando para salir de ella. La madre la toma en brazos para regresar hacia el living comedor. 
FIGURA 7.

LUEGO DE LA SIESTA, ANA BUSCA A MARTINA Y LA CONDUCE EN BRAZOS AL LIVING-COMEDOR.

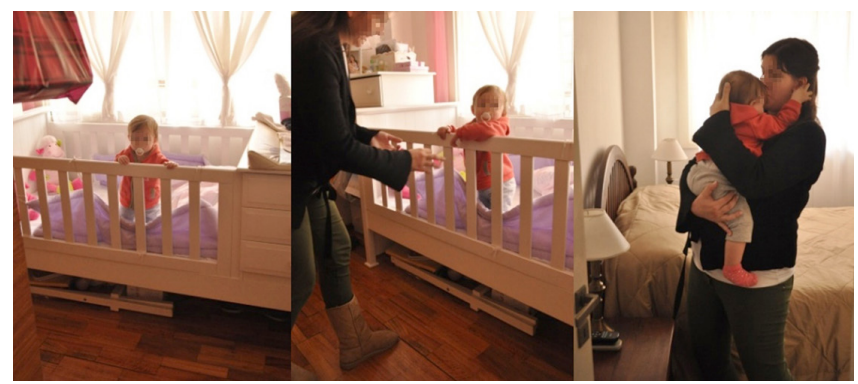

FUENTE: elaboración propia en base a observación de campo.

Interacción: se trata de una escena breve, en la que la madre cumple funciones principalmente de contención y transporte, y Martina no emprende acciones por su cuenta, más que el despertar y el dejarse llevar en brazos de su madre.

Sentimientos: luego de la siesta, la madre y la niña recuperan su ánimo de inicio de mañana, donde gobierna una mayor tranquilidad, y la irritabilidad de Martina previa a la siesta ya no está presente. La madre la contiene afectuosamente, mientras Martina no está aún del todo activa.

\section{Almuerzo, comida - 12:15 a 12:45}

Mientras Martina repuso brevemente su instancia de juego, Ana prepara para la niña una papilla para comenzar a servirle el almuerzo (Figura 8).

Actividad: Ana sienta a Martina junto a ella, y le facilita sucesivamente las cucharadas de comida en su boca. Si bien Martina hace intentos de hacerse de la cuchara, su madre evita que esto ocurra y el plato se consume con bastante rapidez. Por momentos Martina es acomodada en la falda de la madre para dirigir su atención hacia la comida. 
FIGURA 8.

LUEGO DE LA SIESTA, COMIENZA EL ALMUERZO

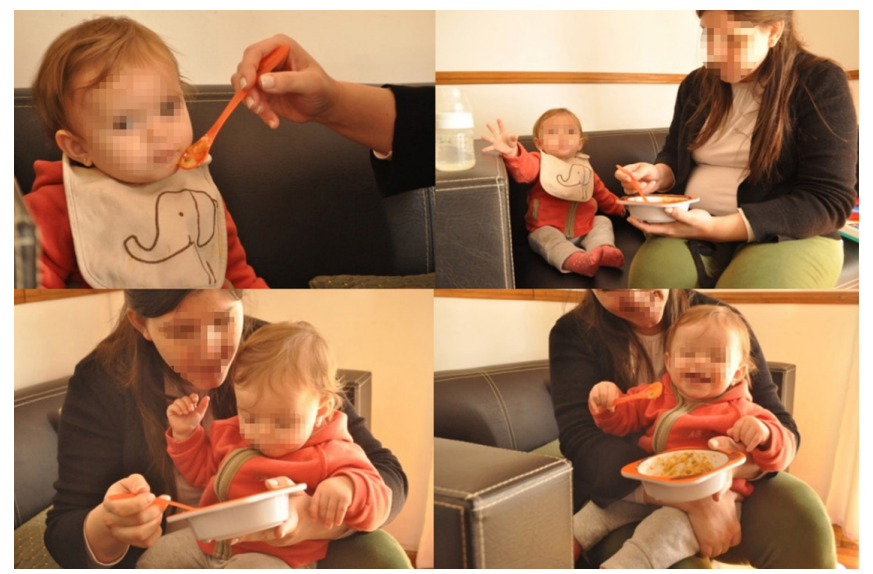

FUENTE: elaboración propia en base a observación de campo.

Interacción: la secuencia ocurre bajo una gran proximidad entre la madre y la niña. Ana procurar evitar una mayor distancia para impedir distracciones de Martina. Con el foco en la comida, la madre le acerca cucharadas a intervalos más o menos regulares, los cuales Martina acepta e ingiere de buen grado.

Sentimientos: a diferencia de la comida sólida del desayuno, el almuerzo ocurre con un bajo grado de autonomía de Martina. No obstante, la comida parece venir a satisfacer un hambre presente, por lo que la velocidad que la madre imprime a la acción parece un ritmo compatible con la disposición de la niña para comer. En un clima de relativa seriedad, ocurren a la par algunos juegos corporales de la madre que permiten distender el clima del almuerzo.

\section{Postre - 12:45 a 13:00}

Transcurridos algunos minutos luego de la papilla, la madre prepara un puré de frutas para ofrecérselo a Martina como postre (Figura 9).

Actividad: mientras Martina juega en el sillón o en el suelo, Ana procura acercarle las cucharadas de postre. Martina, ya sin tanto apetito, responde irregularmente a estos ofrecimientos. 
FIGURA 9.

MARTINA COMPLETA EL ALMUERZO CON UNA PAPILLA DE FRUTAS COMO POSTRE.

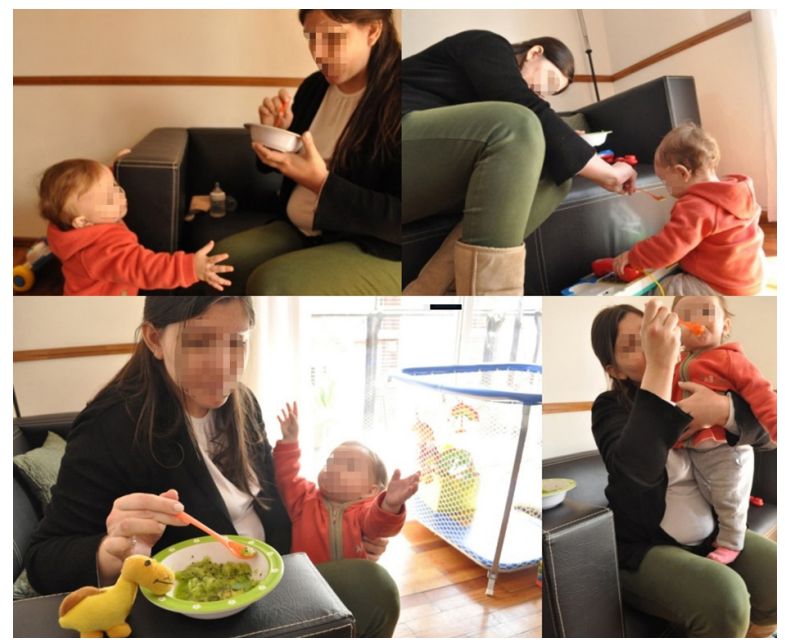

FUENTE: elaboración propia en base a observación de campo.

Interacción: si bien la estructura de la interacción es similar a la de la comida principal, el cambio en el interés de Martina — junto a la menor preocupación de Ana que el carácter accesorio del postre suscita— introducen modificaciones en la interacción. Martina se encuentra con mayor libertad de movimiento, y Ana la busca a intervalos irregulares para ofrecerle un poco más de fruta.

Sentimientos: en comparación a la comida principal, los sentimientos ahora han perdido intensidad. Martina guarda un menor interés en el alimento ofrecido, al tiempo que Ana parece haber perdido cierta firmeza y redirige su acción dando mayor acceso al juego y a tomar con humor el relativo éxito del procedimiento.

\section{Cambiada - 13:00 a 13:15}

Poco después de completado el almuerzo, Ana advierte que el pañal de Martina requiere ser sustituido. Esta situación conduce a regresar nuevamente a la habitación para hacer uso del cambiador que está sobre la cuna. Junto a él se encuentran los pañales de reemplazo, las toallitas y el óleo calcáreo, con los que se higienizará a Martina (Figura Io). 
Actividad: la actividad se compone de varios tramos, en los cuales se hace visible la necesidad de que Martina se mantenga en el espacio del cambiador. Esto es prioritario tanto para poder quitar y volver a poner sus pantalones, como para evitar que el contenido del pañal se derrame fuera del mismo antes de poder desecharlo ya cerrado. Hacia el final de la secuencia, mientras Martina manipula un cepillo y se peina, Ana prepara y le da por vía oral un suplemento prescripto de rutina por su pediatra.

FIGURA 10.

ANA REEMPLAZA LOS PAÑALES DE MARTINA EN EL CAMBIADOR DE SU PIEZA.

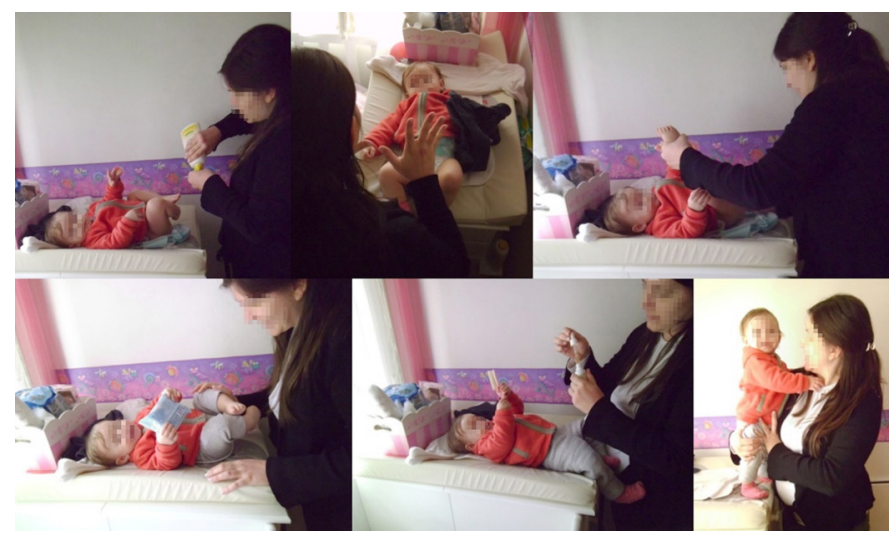

FUENTE: elaboración propia en base a observación de campo.

Interacción: La interacción se produce en esta secuencia a través de varios canales, de modo que mientras varias acciones suceden en la zona higienizada (remoción del pantalón, preparación de elementos, limpieza de la zona) otras tienen lugar en el plano del diálogo entre Martina y Ana, quien se mantiene hablándole y cantando durante el proceso.

Sentimientos: el tono emotivo de esta secuencia es complejo. Ana establece una dinámica de juego, y se entrevé que el sostener un estado de tranquilidad en Martina resulta prioritario. Limpiar su cola puede producir un rechazo a mantenerse recostada, riesgo que Ana busca controlar sosteniendo un diálogo continuo y propiciando momentos de juego visual y musical. Estos juegos parecen buscar 
compensar en cierto modo las molestias que el procedimiento puede provocarle a la niña, pero también da cuenta de las imaginables dificultades que conllevaría completar el proceso sin su cooperación. El juego, cabe sin embargo aclarar, dista mucho de ser una mera estrategia de distracción, y cuando es efectivo logra momentos plenamente alegres para ambas. En la madre, se sostiene una actitud a la vez alerta, decidida y amistosa.

\section{Llama papá - 13:15 a 13:30}

Antes de finalizarse el período de observación, ingresa un llamado telefónico del padre. Se mezclan durante el llamado varios elementos de las secuencias anteriores: juegos corporales y cruces de mirada para entretener a la bebé mientras la madre conversa, alegría compartida por la presencia telefónica del padre y participación de ambas en la conversación (Figura II).

Actividad: Martina está en la falda de Ana mientras tiene lugar la comunicación telefónica. Mientras la madre conversa con el padre, Martina se entretiene primero con un muñeco de peluche que tiene en sus manos y luego busca alcanzar el teléfono celular en uso por su madre con relativo éxito.

ANA Y MARTINA CONVERSAN CON EL PADRE, QUIEN ESTÁ EN SU OFICINA.

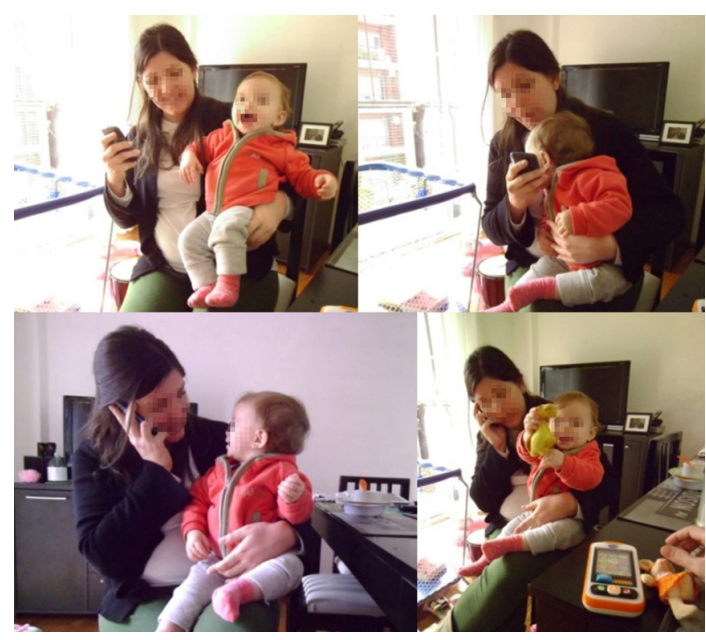

FUENTE: elaboración propia en base a observación de campo. 
Interacción: con la aparición del padre, la interacción gira en torno a una tríada y se alternan el uso del teléfono y el juego, ya sea que esté hablando Ana y aguardando Martina, o que Ana le acerque a Martina el teléfono para escuchar a través de él la voz de su padre.

Sentimientos: un elemento que parece proveer de una emotividad especial a esta secuencia —además de la intensidad del vínculo de quien llama con los demás participantes- es el hecho de que abre una conexión con el exterior de la casa, y con el mundo adulto. En la conversación de Ana con su marido se reponen temas generales de la ciudad y la familia y se proyectan cuestiones organizativas del resto de la jornada.

\section{DISCUSIÓN}

La presente investigación se propuso indagar cómo transcurría la convivencia con niños pequeños en hogares de sectores medios profesionales en la Ciudad de Buenos Aires. Las dinámicas de estos tiempos de cuidado habían sido descriptas en términos ambiguos y a veces contradictorios en entrevistas previas, dando cuenta de una escasez del tiempo, pero a la vez cargados de un «no hacer nada», de lo alegre pero caótico del proceso, de lo fatigante y a la vez reconfortante, describiéndose dificultades para resolver algunas cuestiones prácticas de la madre como el bañarse o el responder mensajes a lo largo de un día normal.

El modelo sugerido por Hommans (actividad, relaciones, sentimientos), organizado aquí en secuencias, nos condujo a poner en evidencia cómo a lo largo de tan solo cuatro horas de cuidado no hubo una actividad (sino muchas), no hubo un sentir (sino muchos), no hubo una modalidad de interacción entre la madre y la bebé (sino muchas).

Antes de analizar este carácter heterogéneo de lo observado, referiremos en primer lugar a un aspecto que conectó todas estas secuencias, y es el carácter continuo y dialógico del vínculo entre Ana y Martina como elemento particular que se mantuvo estructurando la alternancia del amplio número de secuencias observadas.

Este lazo dialógico de sostén continuo (Ana disponible a Martina, Martina visible a Ana), pasó por matices mayormente propios de las diferentes actividades que fueron sucediéndose. En este sentido, las actividades intercalaban necesidades y voluntades de la mamá y la bebé: la organización de las comidas fue en parte 
dispuesta por la madre, pero era también parcialmente modulada por el apetito de la hija; la irritabilidad por el sueño era una marca de la hija, interpretada por la madre; la distracción para lavar unos platos o anticipar la preparación del almuerzo eran iniciativas de la madre que atravesaban las acciones de la nińa. La emotividad pareció así darse en ambas como una superposición de sensaciones: sobre el placer del sostén continuo se desplegaba la incomodidad de un cambio de pañal, y sobre ella, la gracia de un juego distractivo.

Si podemos hablar de copresencia en el tiempo que pasaron Ana y Martina no es porque hayan estado ambas siempre en el mismo espacio (living, cocina, habitación) sino, al contrario, porque más allá de salirse una del campo visual de la otra, o dormir una en la habitación mientras la otra estaba en el living, las dos elaboraban su existencia en la referencia y la certidumbre de la presencia de la otra. No había para ninguna de ambas, en ningún momento, indeterminación sobre la ubicación o la presencialidad de la otra ${ }^{6}$.

El carácter dialógico de la vivencia observada de cuidado surge como un rasgo particular organizador del proceso. El cuidado infantil puede dar pie a una interacción -en otros contextos (ROGOFF, MORELLI Y CHAVAJAY, 2OIO; WHALEY ET AL., 2002) — que incorpore más actores y modalidades. Estas variaciones pueden darse tanto en la pluralidad de cuidadores como de receptores del cuidado, así como en la forma en que quien facilita el cuidado interviene para dar solución a las necesidades que este suscite. En este caso - y fue similar en la mayor parte del tiempo descripto por otras madres de sectores medios profesionales en las entrevistas - el cuidado tuvo como formato estable la díada madre-hija en el contexto de la vivienda como espacio cerrado en lo físico y en lo social.

En segundo lugar, se ha señalado en la introducción un rasgo saliente del vínculo invisible entre lo profesional y lo familiar que operaba en este y otros hogares. El mismo consistía en situar la vivencia del cuidado de día completo sostenido por la madre como una fase provisoria en la organización familiar. Un segundo rasgo de este vínculo emerge del análisis y remite a la singularidad del ritmo observado (la organización temporal de tareas y emociones) si es comparada con otras actividades urbanas en cierto modo más estables como el estudio y la vida profesional.

6] El matiz de estos arreglos en el vínculo entre cuidador y niño han sido abordados en forma comparada para diferentes culturas desde trabajos en antropología y psicología comparada (ver por ejemplo WHALEY, SIGMAN, BECKWITH, COHEN Y ESPINOSA., 2010). 
La secuencia de alteraciones en el contenido de las actividades, en el tipo de relación y en la apuesta emocional de cada una de ellas conforman un horizonte de ritmo cambiante en el tiempo observado. Este dinamismo aparece como un elemento del sistema de interacción creado en el caso de Ana y Martina para coordinar su sostén afectivo (instalado como permanente, pero nada inmóvil) y dar resolución a las necesidades diarias de ambas (de aseo personal y del hogar, de alimento, de juego, de reposo).

Desde la mirada de estas temporalidades, cada secuencia mostró alteraciones respecto a la anterior en la rapidez-lentitud, en el lugar de la madre y la niña en los sincronismos y "postas», en el nivel de pasividad o actividad de cada una de ellas en la acción en que estaban coordinadas. Comer juntas sin apuros trozos de budín, dar una a la otra cucharadas de comida hasta acabar, arrullar a Martina meciéndose de pie, contemplar el juego reponiendo objetos. Esta heterogeneidad de velocidades y alternancias confirma la pertinencia de observar la temporalidad de las prácticas de cuidado, mostrando sin embargo una diversidad mayor que la consignada en la literatura (BESSIN, 20I4). El cuidado se abre en tiempos y velocidades heterogéneas, muy significativas sin embargo para su éxito o su fracaso.

A diferencia del modo en que ocurren las actividades profesionales y en espacios de la educación formal, donde las tareas se asignan por lo general individualmente, se serializan (se hacen una tras la otra) y son extensas (pueden durar horas), la acción Ana-Martina se dio en la simultaneidad y en una modulación compleja de la velocidad y la sincronización ${ }^{7}$.

Si viéramos en esta alternancia de actividades y ritmos algo propio del estar entre adultos de clase media profesional y bebés en la Ciudad de Buenos Aires, resalta el contraste con los órdenes más típicos de los campos laborales y de la educación formal de este sector social. El cuidado —o ciertas formas del cuidar y del estar con bebés - parecieron presentarse con características poco afines al continuum de experiencias educativas-laborales. De ser así, su puesta en práctica puede previsiblemente desconcertar, extrańar, pero sobretodo, demandar tiempo para reorganizarse, comprender y procesar dinámicas de la interacción hasta entonces infrecuentes.

7] Les secuencias 1 , 4, 5, 9, 11 muestran de manera manifiesta la atención en paralelo por parte de la madre, y la brevedad de las secuencias vuelve la experiencia distante al modo de «trabajo concentrado» de larga duración. 
El sistema de interacción (el cual coordina las actividades, interacciones y sentimientos de las diferentes secuencias), que seguramente mutó a lo largo de los meses y que gradualmente pudo ser comprendido e intervenido por ambas hasta llegar al esquema de consensos y códigos que encontramos en el momento de la observación, es dinámico y se codetermina con el marco material, interaccional y simbólico en que trascurre. Estar en un departamento, haber solo un adulto y ningún otro niño, tener II meses de vida, consultar al pediatra, gatear, disponer de heladera y microondas, parecen algunos de los muchos elementos configuratorios que Ana y Martina tomaron como insumos para elaborar sus órdenes prácticos y sus entendimientos.

En el nivel de las actividades, mientras que la simultaneidad del cuidado precisaría compatibilizarse con hábitos de serialización de tareas, vemos también cómo la diversificación de habilidades requeridas (cocinar, jugar, cantar, cambiar, diagnosticar, arrullar, alimentar, pasear) contrasta con la educación por la especialización académica.

En el nivel de la interacción y la afectividad, las dinámicas dialógicas de acompañamiento, de disponibilidad, de espera, se oponen a modos de acción autónomos y autorreferenciales, típicos de la acción de orden más instrumental y profesional ${ }^{8}$.

Sin embargo, no sería adecuado reconocer solamente un carácter pasivo en la interacción de estas lógicas, donde el cuidado se opondría con su temporalidad multimodal y privada a la secuencialidad profesional y pública. La experiencia de la inmersión en la maternidad, como una pausa en la trayectoria profesional, también mostró ser permeable a la instrumentación de saberes y éticas técnicas.

En el caso de Ana y Martina, así como en otros hogares visitados, varios elementos del modo en que era asumido y organizado el cuidado del bebé en el hogar daban cuenta de que la ética práctica profesional también interpela las prácticas de cuidado. En las acciones diarias emergían, sincréticamente, elementos que encarnaban el impulso por la planificación, la mitigación de riesgos, la productividad y la especialización. Este sincretismo secular se materializaba en agendas diarias de actividades para la casa, en la compra anticipada de insumos, en la medicación preventiva, en la producción semanal de papillas y postres para su congelación y administración diaria, entre otros recursos.

8] Adicionalmente, en términos de metas y recompensas, se abre en el cuidado familiar una dinámica donde los resultados fallan con enorme frecuencia (a veces no come, a veces no duerme, a veces se enferma) y cuando las acciones son exitosas, no se destacan del proceso continuo del sostenimiento de la vida, en ausencia de rituales de reconocimiento público tan frecuentes en espacios educativos y profesionales. 


\section{CONCLUSIONES}

En el trabajo de campo se ha podido observar una diversidad de actividades y tipos de interacción entre la bebé y su madre. Las 4 horas de observación derivaron en II secuencias independientes, las cuales dieron cuenta del carácter variado y alternado de la actividad de cuidado infantil en un contexto doméstico de estratos medios profesionales.

Pudo observarse un estilo de cuidado apoyado en el sostén continuo y de vínculo exclusivo con la bebé, basado en una dinámica temporal discordante con los patrones de serialización, especialización, individualidad y autonomía que pueden identificarse como ordenadores de otros niveles prácticos tal como el ejercicio profesional o la socialización educativa.

Estos cruces entre lo conocido y lo nuevo, entre lo organizado y lo por venir, parecen poder comenzar a responder las preguntas que iniciaron el análisis. Dar cuenta, al menos provisoriamente, de las conexiones que puedan ayudar a hacer tangibles los relatos de madres de sus vidas con bebés, a acercar aquellas experiencias donde se unían y acompañaban los límites de la satisfacción y el conflicto, del cansancio y la alegría, del extrañamiento y la comprensión.

\section{Referencias bibliográficas}

ABELS, MONICA; KELLER, HEIDI; PRERNA, MOHITE; de la emoción y la emoción en la sociología», en: HINA, MANKODI; JIISHA, SHASTRI; SHRUTI, BHARGAVA; SUCHETA, JASRAI Y ARUNA LAKJANI (2005): «Early socialization contexts and social experiences of infants in rural and urban Gujarat, India», en: Journal of Cross-Cultural Psychology, 36, pp. 717-738.

ADAM, JEAN-MICHEL (1992): Les textes: tvpes et prototypes, Paris, Nathan.

ALLPORT, GORDON (1977 [1954]): La naturaleza del prejuicio, Buenos Aires, EUDEBA.

ARIZA, MARINA (2015): «La sociología de las emociones como plataforma de la investigación social», en: Emociones, afectos y sociología. Diálogos desde la investigación social y la interdisciplina editado por Ariza, Marina, México D.F., UNAM. BERICAT ALASTUEY, EDUARDO (2000): «La sociología Papers, 62, pp. 145-176.

BERICAT ALASTUEY, EDUARDO (2012): «Emociones», en Sociopedia, ISA, pp. 1-13.

BESSIN, MARC (2014): «Présences sociales: une approche phénoménologique des temporalités sexuées du care», en: Temporalités, 20 (2). BLOCK, JACK (1957): «Studies in the phenomenology of emotions», en: The Journal of Abnormal and Social Psychology 54(3), pp. 358-363.

BOUDON, RAYMOND (1981): La lógica de lo social. Introducción al análisis sociológico, Madrid, Ediciones RIALP.

BRONFENBRENNER, URI (1982): «Children and Families: The Silent Revolution», en: Australian Journal of Sex, Marriage and Family, 3 (3), pp. 111-123. 
CASTAÑEDA RENTERÍA, LILIANA IBETH (2019): «¿Nuevas sujetas, nuevas identidades? La vivencia profesional en la configuración de la identidad de género", en: Nóesis. Revista de Ciencias Sociales y Humanidades, 28 (55), pp. 88-108.

CHAHBENDERIAN, FLORENCIA (2013): «Disciplina: iestás ahí? Algunas reflexiones del amor a las reglas en torno a Émile Durkheim», en: Teoría social, cuerpos y emociones compilado por Adrián Scribano. Buenos Aires, Estudios Sociológicos Editora. COLANGELO, MARÍA ADELAIDA (2006): «La crianza en disputa. Un análisis del saber médico sobre el cuidado infantil», en: Simposio no. 22: Niñez y juventud: Perspectivas en disputa y abordaje etnográfico, Universidad Nacional de La Plata.

COVARRUBIAS FEREGRINO, ARLETTE (2018): «Poder, normas sociales y desigualdad de las mujeres en el hogar», en: Nóesis. Revista de Ciencias Sociales y Humanidades, 27 (53), pp. 140-158. CRUZATA SANTOS, RUBÉN (2005): «Aproximación sociológica a los estudios de la familia: escuelas, conceptos y tendencias», en: Ecuador Debate. Dimensiones de la cuestión regional, 65, pp. 223-234.

DE GRANDE, PABLO (2015): «Cambios y continuidades en los vínculos interpersonales de sectores medios urbanos tras la llegada de un bebé», en: Horizontes Sociológicos, 6 (3), pp. 73-88.

DE GRANDE, PABLO (2016): «Diseñado para bebés. Objetos y prácticas en el primer año de vida», en Revista Latinoamericana de Ciencias Sociales, Niñez y Juventud, 14 (1), pp. 287-300.

DETTANO, ANDREA (2013): "Cuerpos y emociones en la teoría social clásica: Georg Simmel», en: Teoría social, cuerpos y emociones, compilado por Adrián Scribano, Buenos Aires, Estudios Sociológicos Editora.

FAUR, ELEONOR (2012): «El cuidado infantil desde las perspectivas de las mujeres-madres. Un estudio en dos barrios populares del Área Metropolitana de Buenos Aires», en: Las lógicas del cuidado infantil. Entre las familias, el Estado y el mercado, editado por V. Esquivel, E. Faur y E. Jelin, Buenos Aires, IDES.

FISHER, BERENICE Y TRONTO, JOAN (1991): «Toward a feminist theory of care», en Circles of Care: Work and Identity in Women's Lives, editado por ABEL E. Y NELSON M., ALBANY (Nueva York), State University of New York Press.

FLORES ÁNGELES, ROBERTA LILIANA Y GUERRERO, OLIVIA TENA (2014): «Maternalismo y discursos feministas latinoamericanos sobre el trabajo de cuidados: un tejido en tensión», en: Iconos. Revista de Ciencias Sociales, 50, pp. 27-42.

GILLIGAN, CAROL (1982): In a Different Voice, Cambridge (EE. UU.), Harvard University Press. GILLIGAN, CAROL (2013): «La resistencia a la injusticia: una ética feminista del cuidado», en: Cuadernos de la Fundació Víctor Grífols, 30, pp. 40-67.

GUTIÉRREZ SASTRE, MARTA (2002): «Triangular público, doméstico y privado, o ¿cómo negociar en pareja?», en: Reis. Revista Española de Investigaciones Sociológicas, 99, pp. 61-85.

HAGESTAD, GUNHILD Y UHLENBERG, PETER (2005): «The Social Separation of Old and Young: A Root of Ageism», en: Journal of Social Issues, 61(2), pp. 343-360.

HOCHSCHILD, ARLIE (1975): «The sociology of feeling and emotion: Selected possibilities», en: Sociological Inquiry, 45(2-3), pp. 280-307.

HOCHSCHILD, ARLIE Y MACHUNG, ANNE (1989): The Second Shift, Nueva York, Penguin.

HOMANS, GEORGE (1970): El Grupo humano, Buenos Aires, EUDEBA, pp. 52-74.

LUNA ZAMORA, ROGELIO (2000): «Introducción a la Sociología de las Emociones», en: Revista Universidad de Guadalajara, 18. 
MAFFÍA, DIANA (2007): "Epistemología feminista: La subversión semiótica de las mujeres en la ciencia», en: Revista Venezolana de Estudios de la Mujer, 12 (28), pp. 63-98.

MILGRAM, STANLEY (1963): «Behavioral Study of Obedience», en: Journal of Abnormal and Social Psychology, 67 (4), pp. 371-378.

MUÑIZ GALLARDO, ERIKA Y MARÍA ELENA RAMOS TOVAR (2019): «La presión social para ser madre hacia mujeres académicas sin hijos», en: Nóesis. Revista de Ciencias Sociales y Humanidades, 28 (55), pp. 64-87.

NARI, MARCELA (2004): Políticas de la maternidad y maternalismo político: Buenos Aires (18901940), Buenos Aires, Biblos.

PETIT, EMMANUEL (2013): «L'économie du comportement et la théorie du care. Les enjeux d'une filiation», en: Revue du MAUSS, 41.

POWELL, CHRISTOPHER Y DEPELTEAU, FRANÇOIS (EDS.)

(2013): Conceptualizing Relational Sociology, Nueva York, Palgrave Macmillan.

PRINZ, JESSE (2004): «Which emotions are basic», en: Emotion, evolution, and rationality, 69, pp. 88-106.

ROGOFF BARBARA, GILDA MORELLI Y PABLO CHAVAJAY (2010): «Children's Integration in Communities and Segregation from People of Differing Ages», en: Perspectives on Psychological Science, 5, pp. 431-440.

ROGOFF, BARBARA (1981): "Adults and peers as agents of socialization: A highland Guatemalan profile», en: Ethos, 9 (1), pp. 18-36.

SÁNCHEZ MARTÍNEZ, ALICIA VERÓNICA (2002): «La macrooperación descriptiva: sus operaciones lógico-discursivas», en: Cuicuilco, 9 (24).
SCRIBANO, ADRIÁN (ED.) (2015): Los estudios sociales sobre cuerpos y emociones en Argentina: un estado del arte, Buenos Aires, Estudios Sociológicos Editora.

SCRIBANO, ADRIÁN (2012): «Sociología de los cuerpos/emociones», en: Revista Latinoamericana de Estudios sobre Cuerpos, Emociones y Sociedad, 10, pp. 93-113.

SEGATO, RITA (2018): Contra-pedagogías de la crueldad, Buenos Aires, Prometeo.

SHEETS JOHNSTONE, MAXINE (1990): The Roots of Thinking, Philadelphia, Temple University Press. SHILLING, CHRIS (2003): The Body and Social Theory, Londres, Sage.

TENOUTEN, WARREN (2006): A general theory of emotions and social life, Nueva York, Routledge THOITS, PEGGY (1989): «The Sociology of Emotions», en: Annual Review of Sociology, 15, pp. 317-342.

TICINETO CLOUGH, PATRICIA Y JEAN HALLEY (2007): The affective turn, Durham (Estados Unidos), Durham University Press.

TRONTO, JOAN (1993): Moral Boundaries. A Political Argument for an Ethic of Care, Londres, Routledge.

TRONTO, JOAN (2008): «Du care», en: Revue $d u$ MAUSS, 32, pp. 243-265.

TRONTO, JOAN (2013): Caring Democracy, Nueva York, New York University Press.

WHALEY SHANNON, SIGMAN, MARIAN; BECKWITH, LEILA; COHEN, SARALE Y ESPINOSA, SARALE (2002): «Infantcaregiver interaction in Kenya and the United States: The Importance of multiple caregivers and adequate comparison samples», en: Journal of Cross-Cultural Psychology, 33(3), pp. 236-247. 